\title{
Emotional content impacts how executive function ability relates to willingness to wait and to work for reward
}

\author{
Katherine S. F. Damme ${ }^{1}$ (D) - Nicholas J. Kelley ${ }^{1} \cdot$ Meghan E. Quinn ${ }^{1,2}$ • James E. Glazer ${ }^{1} \cdot$ Iris Ka-Yi Chat ${ }^{1,3}$. \\ Katherine S. Young ${ }^{4,5} \cdot$ Robin Nusslock $^{1} \cdot$ Richard Zinbarg $^{1,6} \cdot$ Susan Bookheimer ${ }^{4} \cdot$ Michelle G. Craske $^{4}$
}

Published online: 1 April 2019

(C) The Psychonomic Society, Inc. 2019

\begin{abstract}
Research has demonstrated that better value-based decision making (e.g., waiting or working for rewards) relates to greater executive function (EF) ability. However, EF is not a static ability, but is influenced by the emotional content of the task. As such, EF ability in emotional contexts may have unique associations with value-based decision making, in which costs and benefits are explicit. Participants $(N=229)$ completed an EF task (with both negative and neutral task conditions) and two value-based decision-making tasks. Willingness to wait and to work were evaluated in separate path models relating the waiting and working conditions to the $\mathrm{EF}$ conditions. Willingness to wait and willingness to work showed distinct relationships with $\mathrm{EF}$ ability: Greater EF ability on a negative, but not on a neutral, $\mathrm{EF}$ task was related to a willingness to wait for a reward, whereas greater EF ability across both EF tasks was related to a greater willingness to work for a reward. EF ability on a negative EF task showed an inverted-U relationship to willingness to wait for reward, and was most related to willingness to wait at a 6-month delay. Greater $\mathrm{EF}$, regardless of whether the task was negative or neutral, was related to a greater willingness to work when reward was uncertain $(50 \%)$ or was likely $(88 \%)$, but not when reward was unlikely $(12 \%)$. This study suggests that the emotional content of valuebased decisions impacts the relationship between EF ability and willingness to wait or to work for reward.
\end{abstract}

Keywords Executive function $\cdot$ Decision making $\cdot$ Temporal discounting $\cdot$ Effort discounting $\cdot$ Reward $\cdot N$-back $\cdot$ EEfRT $\cdot$ DDT

The best decision is not always the easiest one. The best decision can be a particularly difficult choice when that decision requires an individual to wait (Chung \& Herrnstein, 1967) or work (Treadway, Buckholtz, Schwartzman, Lambert, \& Zald, 2009) for a reward, because of the cost of time or effort expended. In contrast, easier decisions often do not require waiting or working for a reward. These cost-benefit dilemmas may lead individuals to make decisions that are not in their long-term interest, from how they spend their money to how

Katherine S. F. Damme

Kate.Damme@u.northwestern.edu

Northwestern University, Evanston, IL, USA

2 Vanderbilt University, Nashville, TN, USA

3 Temple University, Philadelphia, PA, USA

4 University of California, Los Angeles, Los Angeles, CA, USA

5 King's College, London, UK

6 Family Institute at Northwestern University, Evanston, IL, USA they spend their time. These value-based decisions can have economic costs for both individuals and society, from retirement (Laibson et al., 1998) to obesity (Wolf \& Colditz, 1998) to academic achievement (Gatzke-Kopp, Ram, Lydon-Staley, \& DuPuis, 2018). Therefore, it is critical to understand the cognitive processes underlying value-based decision making.

Value-based decision making refers to decisions for which the costs and benefits of options are explicit (Rangel, Camerer, \& Montague, 2008; Zelazo \& Carlson, 2012). During valuebased decision making, the costs and benefits of each option are compared in order to create a subjective value of that option - that is, the value of a reward accounts for the costs of working or waiting (Rangel et al., 2008). Choices are made on the basis of the subjective value of each option, typically with the goal of maximizing value and minimizing cost. Although research on value-based decision making covers many topics, this article addresses two common forms of value-based decision making: a willingness to wait for reward, and a willingness to work for reward. Despite the general tendency to prefer low effort and immediate rewards, there are substantial individual differences in tendencies toward 
willingness to wait and to work. Such individual differences are linked to a number of behaviors, from drug abuse to depression (Barch, Treadway, \& Schoen, 2014; Odum, 2011a; Treadway et al., 2009).

Willingness to wait for a reward refers to a tendency to forgo smaller immediate rewards for larger future rewards (Odum, 2011a). Individuals generally prefer immediate rewards, and the immediacy of a reward disproportionally impacts decision making (Chung \& Herrnstein, 1967). Behaviorally, a higher willingness to wait for reward may lead to better long-term choices, such as greater saving for retirement (Odum, 2011b). Less willingness to wait has been linked to a number of other real-world behaviors, including increased drug abuse, obesity, and gambling (Daugherty \& Brase, 2010; Kirby, Winston, \& Santiesteban, 2005; Odum, 2011a). Additionally, individual differences in willingness to wait are related to reward sensitivity (Odum, 2011a; Pornpattananangkul \& Nusslock, 2016) and impulsivity (Benningfield et al., 2014; Hariri et al., 2006). Biologically, individual differences in willingness to wait choice tendencies are associated with activity in the anterior frontal cortex (Shamosh et al., 2008), amygdala (Churchwell, Morris, Heurtelou, \& Kesner, 2009), striatum, and posterior insula (Wittmann, Leland, \& Paulus, 2007). Taken together, past research has demonstrated that individual differences in willingness to wait for reward have a variety of real-world implications.

Willingness to work refers to a tendency to expend effort in order to receive a reward (Botvinick, Huffstetler, \& McGuire, 2009; Croxson, Walton, O'Reilly, Behrens, \& Rushworth, 2009). Individuals generally prefer to preserve effort in pursuit of rewards and to weigh the subjective value of rewards against the amount of effort that must be expended to access a reward (Botvinick et al., 2009; Sugiwaka \& Okouchi, 2004). Behaviorally, individual differences in willingness to work are related to reward sensitivity (Barch et al., 2014; Johnson, Swerdlow, Treadway, Tharp, \& Carver, 2017; Treadway et al., 2012), and to functional impairment in clinical populations (Barch et al., 2014; Treadway et al., 2009; Treadway et al., 2012). Biologically, willingness to work for rewards is related to functional activity in the dorsal anterior cingulate cortex, anterior insula, ventral medial prefrontal cortex, and nucleus accumbens, which are all part of the larger value-based decisionmaking network (Aridan, Malecek, Poldrack, \& Schonberg, 2019; Arulpragasam, Cooper, Nuutinen, \& Treadway, 2018; Botvinick et al., 2009; Croxson et al., 2009; Pessiglione, Vinckier, Bouret, Daunizeau, \& Le Bouc, 2017). Overall, research on individual differences in willingness to work has provided insight into clinical symptomatology.

Despite both willingness to wait and willingness to work being implicated in value-based decision making, there is evidence that these two tendencies are dissociable (Gatzke-Kopp et al., 2018; Klein-Flügge, Kennerley, Saraiva, Penny, \& Bestmann, 2015). Behaviorally, tendencies toward willingness to work and to wait are dissociable within an individual, such that someone may be more willing to wait than to work or more willing to work than to wait (Gatzke-Kopp et al., 2018; Klein-Flügge et al., 2015). Additionally, waiting and working for rewards impacts the subjective value of the reward in distinct ways. Waiting for a reward, even at short delays, immediately negatively impacts the value of that reward, whereas working for reward has less impact on the subjective value, but steeply impacts the value of rewards when working for reward becomes tiring (Gatzke-Kopp et al., 2018; Klein-Flügge et al., 2015).

Biologically, the tendencies toward willingness to wait and willingness to work for rewards are related to distinct but overlapping neural networks. Human neuroimaging studies have reported that the subjective value of a reward is related to activity in the ventral striatum, pregenual anterior cingulate cortex, and inferior aspects of the frontal cortex for both tasks involving willingness to wait and tasks involving willingness to work (Massar, Libedinsky, Weiyan, Huettel, \& Chee, 2015; Prévost, Pessiglione, Météreau, Cléry-Melin, \& Dreher, 2010; Seaman et al., 2018). However, decisions regarding whether to work or wait for reward are related to activation in distinct areas (Prévost et al., 2010; Seaman et al., 2018). In direct comparisons of waiting and working for rewards, studies have shown that choosing to wait for a reward is related to activity in the ventral medial prefrontal cortex (Massar et al., 2015; Prévost et al., 2010; Rudebeck, Walton, Smyth, Bannerman, \& Rushworth, 2006; Seaman et al., 2018), whereas choosing to wait for a reward is related to activity in the anterior cingulate cortex (Prévost et al., 2010; Seaman et al., 2018). Additionally, behavioral neuroscience research has demonstrated that lesions of the orbitofrontal cortex are related to decreased tendencies toward willingness to wait, but not toward willingness to work (Rudebeck et al., 2006). In contrast, lesions of the anterior cingulate cortex are related to decreased willingness to work, but not to decreased willingness to wait (Rudebeck et al., 2006). In both studies, individuals demonstrated intact reward valuations but disruptions to decision making, suggesting specific impairments in executive function ability (Gatzke-Kopp et al., 2018; Klein-Flügge et al., 2015; Massar et al., 2015; Prévost et al., 2010; Rudebeck et al., 2006; Seaman et al., 2018).

Although there are critical differences between willingness to wait and to work, both of these tendencies are thought to rely on executive function processes (Bickel, Jarmolowicz, Mueller, Gatchalian, \& McClure, 2012; Hinson, Jameson, \& Whitney, 2003; Olson, Hooper, Collins, \& Luciana, 2007; Shamosh \& Gray, 2008; Sugiwaka \& Okouchi, 2004). Executive function is a broad term for cognitive processes that allow individuals to regulate thoughts and actions in a goaldirected manner and to adaptively respond to dynamic contexts (Banich, 2009; Friedman \& Miyake, 2017; Miyake et al., 2000; Quinn \& Joormann, 2015). In contrast to automatic or highly trained responses, executive function processes allow individuals to flexibly respond to the environment in order to 
achieve a goal. Executive function includes three categories of cognitive processes: updating, inhibition, and shifting (Miyake et al., 2000). Each of these processes is implicated in decision making (Banich, 2009; Friedman \& Miyake, 2017; Rangel et al., 2008). During value-based decision making, an individual must consider the subjective costs and benefits of each option in working memory (updating), must respond to irrelevant information that needs to be inhibited (inhibition), and must switch between mindsets in order to fully consider each option (shifting). Given the potential role of all three of these processes in value-based decision making (Banich, 2009; Rangel et al., 2008) and the high intercorrelations between them (Friedman \& Miyake, 2017; Miyake et al., 2000), we will treat these processes as a unified executive function ability.

Executive function has another feature that may be critical to value-based decision making: Specifically, executive function ability varies across nonemotional (cold) and emotional (hot) tasks (Zelazo \& Carlson, 2012). Cold executive function (EF) tasks refer to executive function that takes place in affectively neutral or nonemotional contexts, whereas hot $\mathrm{EF}$ tasks refer to executive function situated in motivationally or emotionally salient contexts (Zelazo \& Carlson, 2012). In fact, executive function processes are sensitive to distracting emotional information even when that emotionally salient information is not relevant to the task (Joormann \& Vanderlind, 2014; Quinn \& Joormann, 2015; Schmeichel \& Tang, 2015). Additionally, poorer executive function ability on a hot EF task is related to reduced emotion regulation (Hofmann, Schmeichel, \& Baddeley, 2012; Schmeichel \& Tang, 2015), increased problem-focused cognitive strategies (Compton et al., 2011), vulnerability to psychopathology (Joormann \& Quinn, 2014), and increased risk-taking behaviors (Patrick, Blair, \& Maggs, 2008). As a result, executive function ability on a hot EF task may be particularly important in value-based decision making in which emotionally salient costs and rewards are explicit.

Unlike unemotional decisions, value-based decisions inherently contain emotionally salient content, namely the costs of waiting or working and potential rewards. These explicit costs and rewards create a motivational and emotional (hot) context for decisions that involve willingness to wait or to work. These emotional contexts of willingness to wait (Odum, 2011a, 2011b) and to work (Treadway et al., 2012) for reward have led some to theorize that value-based decisions are better characterized by executive function ability in the face of hot, emotional information than by such ability in cold, neutral contexts (Zelazo \& Carlson, 2012). Past research, however, has largely examined the relation of executive function ability on a cold EF task to value-based decision making without considering the role of the emotional context of rewards and costs (Bickel et al., 2012; Hinson et al., 2003; Olson et al., 2007; Shamosh \& Gray, 2008; Sugiwaka \&
Okouchi, 2004). What remains unknown is if value-based decision making relates more to executive function ability in the face of distracting emotional information (i.e., a hot EF task) or neutral information (i.e., a cold EF task).

The purpose of the present study was to assess how individual differences in executive function ability during both hot and cold EF tasks relate to the willingness to wait and to work for rewards. Consistent with past research (e.g., Bickel et al., 2012; Hinson et al., 2003; Olson et al., 2007; Shamosh \& Gray, 2008; Sugiwaka \& Okouchi, 2004), we hypothesized that executive function ability on a cold EF task would positively relate to both a willingness to work and a willingness to wait. In novel analyses, we hypothesized that executive function ability on a hot EF task would also relate positively to both a willingness to wait and a willingness to work. We further predicted that executive functioning on a hot EF task would account for significantly more variance in the tendencies toward willingness to wait and to work than would executive function ability on a cold EF task, because value-based decisions involve distracting emotional, hot information regarding potential costs and rewards (Zelazo \& Carlson, 2012). Finally, we examined the relationships between task conditions involving either willingness to wait or to work and executive function ability. Within the willingness-towait task, we manipulated six delay conditions, and within the willingness-to-work task, we manipulated three different probabilities of receiving a reward: unlikely $(12 \%)$, uncertain $(50 \%)$, or likely $(88 \%)$. We predicted that a positive association between executive function ability and willingness to wait for rewards would be stronger for earlier (vs. later) rewards. Furthermore, we predicted that positive associations between executive function ability and willingness to work for rewards would be stronger for trials with a high likelihood of being rewarded.

\section{Method}

\section{Participants}

The participants in this study were a part of the longitudinal multisite Brain, Motivation, and Personality Development (BrainMAPD) Project, conducted at the University of California, Los Angeles (UCLA), and Northwestern University. Participant recruitment was stratified to include a full range of both reward and threat sensitivity at each site. A total of 282 young adults participated (65\% female, ages 18 21 years); 114 of these participants were recruited from the Los Angeles, California, area and participated at UCLA (65\% female, ages 18-20 years), and 168 were recruited from the Chicago, Illinois, area and participated at Northwestern University (66\% female, ages 18-20 years). At both sites, participants completed an EF task (affective $N$-back task; 
Quinn \& Joormann, 2015) and two value-based decisionmaking tasks in rewarding contexts: both the delaydiscounting task (DDT; M. W. Johnson \& Bickel, 2002; Lagorio \& Madden, 2005) and the "effort expenditure for rewards" task (EEfRT; Treadway et al., 2009). Across all tasks, a total of 53 participants were excluded on the basis of performance criteria established in the literature for each respective task: five for low accuracy in the $N$-back, 27 for DDT performance that included inconsistent choices, 26 for EEfRT performance that included not completing enough trials, and five for poor performance across multiple tasks. For each task, the specific exclusion criteria are discussed in detail below. Analyses were conducted on the 229 remaining participants with acceptable data on all three tasks. The participants' data are summarized in Table 1.

\section{Executive function task}

$\mathrm{N}$-back tasks measure the accuracy with which participants update their working memory, a component of executive function (Chatham et al., 2011; Kirchner, 1958; Quinn \& Joormann, 2015). In the affective $N$-back task, participants completed 240 trials that consisted of a word presented for $500 \mathrm{~ms}$, followed by a blank screen presented for $2,500 \mathrm{~ms}$ (Quinn \& Joormann, 2015). For each word displayed, participants were instructed to indicate whether the word matched the one that had been presented two trials previously, as quickly and accurately as possible. If the currently displayed word matched the word displayed two trials previously, participants were instructed to press a key labeled "yes." If the word did not match, they were instructed to press a key labeled "no." This procedure was completed across two conditions (neutral and negative) with 120 trials each. The neutral condition contained only neutral words (e.g., "curtains"), and the negative condition contained both negative (e.g., "failure") and neutral words (the word choices are described in Quinn \& Joormann, 2015). These conditions appeared in randomized order in two 60-trial blocks. Our task design is represented in Fig. 1A.

Response accuracy was calculated for each of the negative and neutral conditions (Snyder, Miyake, \& Hankin, 2015). Executive function ability in a hot task condition was defined as accuracy during the negative word condition. Executive function ability in a cold task condition was defined as accuracy during the neutral word condition. Errors were defined as multiple responses (i.e., both "yes" and "no" pressed on a single trial), omitted responses, and incorrect responses. Accuracy was used as a basis for the exclusion for five participants. Participants with accuracy scores more than three standard deviations below the group average accuracy for that neutral $(M=87 \%, S D=10 \%)$ or negative $(M=88 \%, S D=$ $10 \%$ ) conditions were considered outliers. It is notable that all of the outlier scores fell below chance performance $(50 \%)$ in both the neutral $(M=36 \%, S D=7 \%)$ and negative $(M=35 \%$, $S D=9 \%$ ) conditions, which suggests that that poor accuracy was not likely related to condition.

\section{Willingness to wait in the delay-discounting task (DDT)}

The DDT is a temporal-discounting task that assesses willingness to wait for hypothetical rewards (Ahn et al., 2011; Rachlin, Raineri, \& Cross, 1991). For each trial, participants made a series of choices between a smaller immediate reward and a future reward of $\$ 800$ for six delay-period conditions that continued for six trials, for a total of 36 trials. After a choice was made, a square was presented on the screen reflecting the choice for $1,500 \mathrm{~ms}$, followed by a $3,000-\mathrm{ms}$ fixation. Our task design is represented in Fig. 1B. The future choice was set to one of six delay periods: 2 weeks, 1 month, 2

Table 1 Differences by data collection site

\begin{tabular}{|c|c|c|c|c|}
\hline & $\begin{array}{l}\mathrm{NU} \\
M(S t D)\end{array}$ & $\begin{array}{l}\text { UCLA } \\
M(S t D)\end{array}$ & Statistic & $p$ Value \\
\hline Age & $19.26(0.64)$ & $19.04(0.65)$ & $t(227)=2.53$ & $.012^{*}$ \\
\hline Sex & $40 \mathrm{M} / 73 \mathrm{~F}$ & $48 \mathrm{M} / 94 \mathrm{~F}$ & $\chi^{2}(2)=0.90$ & .64 \\
\hline Affective $N$-back accuracy: Neutral & $88.8 \%(0.07 \%)$ & $89.5 \%(0.08 \%)$ & $t(227)=0.75$ & .93 \\
\hline Affective $N$-back accuracy: Negative & $87.4 \%(0.07 \%)$ & $8.88 \%(0.08 \%)$ & $t(227)=1.31$ & .35 \\
\hline DDT $k$ value & $0.23(0.22)$ & $0.16(0.05)$ & $t(227)=1.29$ & .20 \\
\hline EEfRT: Total hard choices & $39.3 \%(14.3 \%)$ & $35.4 \%(16.7 \% 14.2 \%)$ & $t(227)=1.88$ & .07 \\
\hline EEfRT: $12 \%$ proportion of hard choices & $10.7 \%(16.9 \%)$ & $11.3 \%(14.2 \%)$ & $t(227)=0.31$ & .76 \\
\hline EEfRT: $50 \%$ proportion of hard choices & $44.2 \%(25.4 \%)$ & $38.2 \%(22.8 \%)$ & $t(227)=1.88$ & .06 \\
\hline EEfRT: $88 \%$ proportion of hard choices & $57.5 \%(21.1 \%)$ & $57.5 \%(21.1 \%)$ & $t(227)=1.90$ & .06 \\
\hline
\end{tabular}

UCLA = University of California, Los Angeles; NU = Northwestern University; $M=$ mean, $S t D=$ standard deviation. ${ }^{*}$ Comparison that is significant and survives correction for multiple comparisons 


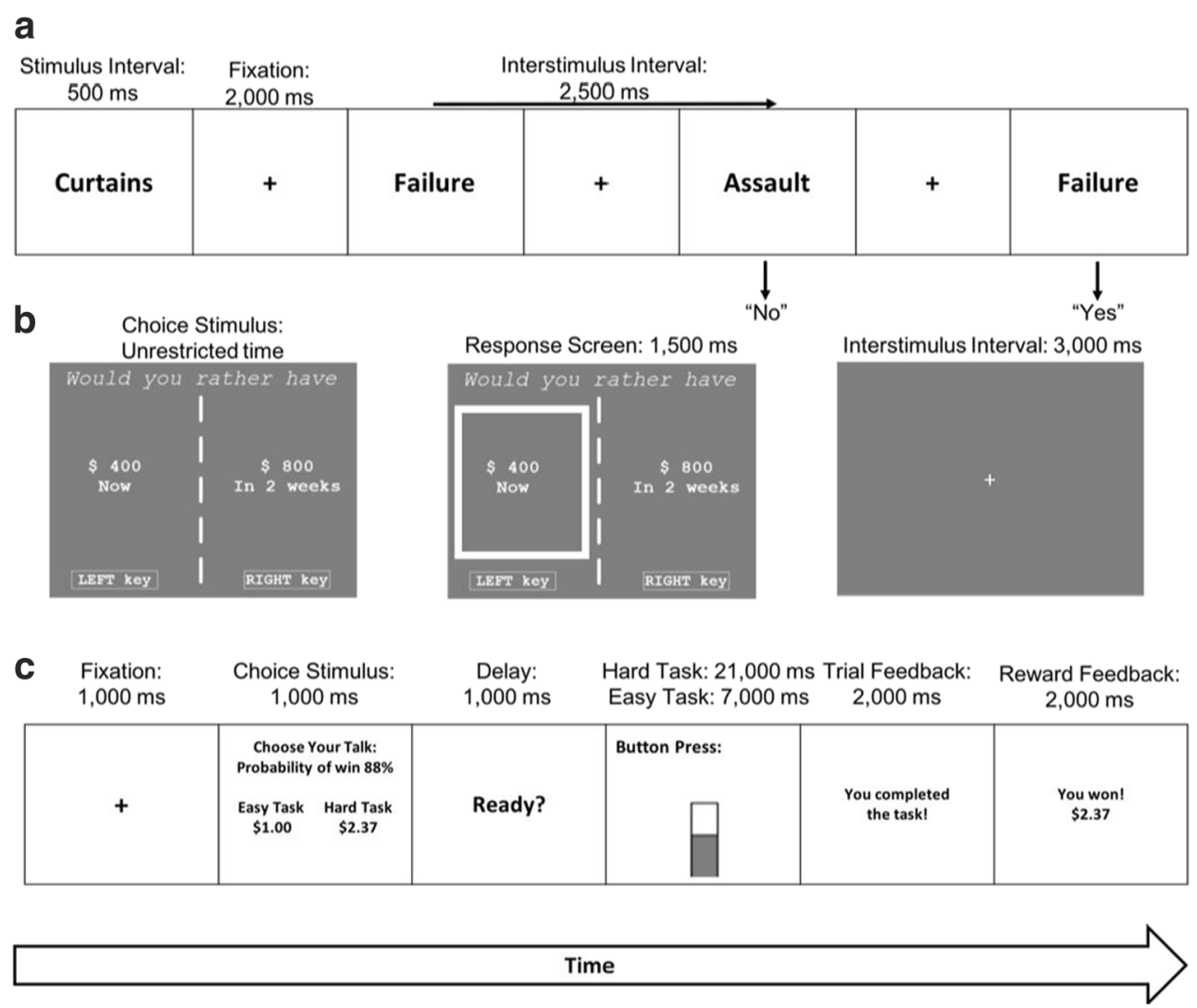

Fig. 1 Task structure and stimuli for the (A) affective $N$-back task, (B) delay-discounting task, and (C) effort expenditure for reward task

months, 6 months, 1 year, 3 years, or 10 years into the future (Ahn et al., 2011; Pornpattananangkul \& Nusslock, 2016; Rachlin et al., 1991). At each delay period, choices began by pitting $\$ 400$ now against $\$ 800$ in the future. If the participant chose the immediate reward, then the immediate reward amount on the subsequent trial decreased by half the distance between that amount and $\$ 0$ (Ahn et al., 2011; Du, Green, \& Myerson, 2002). All decisions were for hypothetical rewards and delays, as previous research had shown similar response patterns, whether the DDT was played for real or hypothetical reward amounts (M. W. Johnson \& Bickel, 2002; Lagorio \& Madden, 2005). For each of the six delay periods, participants completed six trials pitting smaller rewards against $\$ 800$ in the future. On the final trial in each delay-period condition (e.g., 1 month, 1 year, 10 years), the resultant small reward amount was the subjective value of $\$ 800$ at the given delay period. For example, if a participant always chose the smaller amount during the 10-year condition, then $\$ 800$ in 10 years would have a subjective value equal to receiving $\$ 6.25$ immediately.

For each participant, the subjective value of $\$ 800$ at each delay period was fitted into a hyperbolic model; for a groupaverage curve, see Fig. 2D (Pornpattananangkul \& Nusslock, 2016). The steepness of the slope within this hyperbolic model ( $k$ value) reflected the preference for smaller-but-immediate (as compared to larger-but-delayed) rewards, Fig. 2D. The DDT $k$ values were normalized using the natural $\log$ of $k$ as an index of individual differences in delay-discounting tendencies (Shead \& Hodgins, 2009). Participants were excluded on the basis of $R^{2}$ value, which was calculated on the basis of the hyperbolic curve. A low $R^{2}$ suggests that an individual's decisions on the particular task were highly irregular and could not be reliably described by the DDT hyperbolic $k$ model. Additionally, irregular or inconsistent choices on the DDT might reflect either a lack of understanding or a lack of attention to the discounting task. A "low"- $R^{2}$ hyperbolic curve was defined as $R^{2}$ equal to zero or an extreme value (defined by $R^{2}$ at the 25 th percentile - [3*interquartile range]); in this study, the threshold was -.52 , which excluded 21 individuals (Shead \& Hodgins, 2009). Finally, six individuals were excluded for showing no discounting behavior- that is, for always preferring the larger and later reward - since this value cannot be modeled with a hyperbolic curve. Although it is possible that these individuals would always prefer larger but later rewards (a potentially relevant endophenotype), it is also possible that the reward value of $\$ 800$ was not sufficient to detect meaningful behavioral differences in these individuals.

\section{Willingness to work and the "effort expenditure for reward" task (EEfRT)}

The EEfRT is an effort-discounting task that assesses willingness to work for rewards by pitting small rewards 
a

Affective N-Back Accuracy by Condition

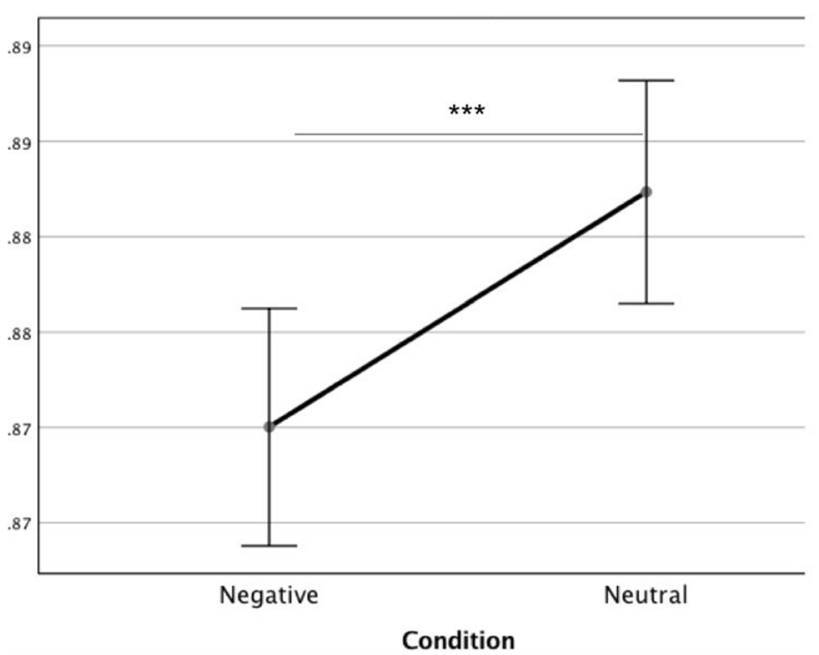

C Proportion of Hard Choices by Reward Probability Condition

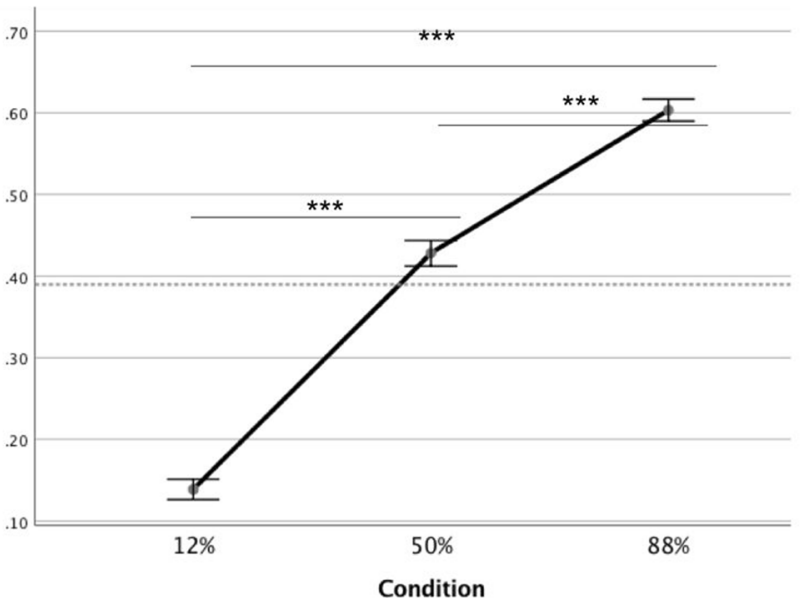

Fig. 2 Main effects of condition in the affective $n$-back task (condition average controlling for the impact of collection site $\mathbf{A}$, the correlation between condition performance B), the "effort expenditure for reward" task $(\mathbf{C})$, and the delay-discounting task (D). The affective $n$-back task showed a small but significant difference in accuracy by condition (A),

requiring minimal physical effort against larger rewards requiring considerable physical effort. In the EEfRT, participants were asked to choose between easy and hard trials. There were three probability-of-reward conditions $(88 \%$, $50 \%, 12 \%$ ), and each trial had a single probability of reward. For all trials, participants made button presses in order to reach a goal number of presses. The participant could view progress toward the goal on the screen. In the easy task, participants were required to make 30 button presses, using the dominant-hand index finger within $7 \mathrm{~s}$, for a potential $\$ 1.00$ reward. In the hard task, participants were required to make 100 button presses using the nondominant little finger in $21 \mathrm{~s}$, for a variable reward magnitude (\$1.24-\$4.30). For the hard task, each combination of probability and reward value appeared once. All trials were presented in a consistent, randomized order.

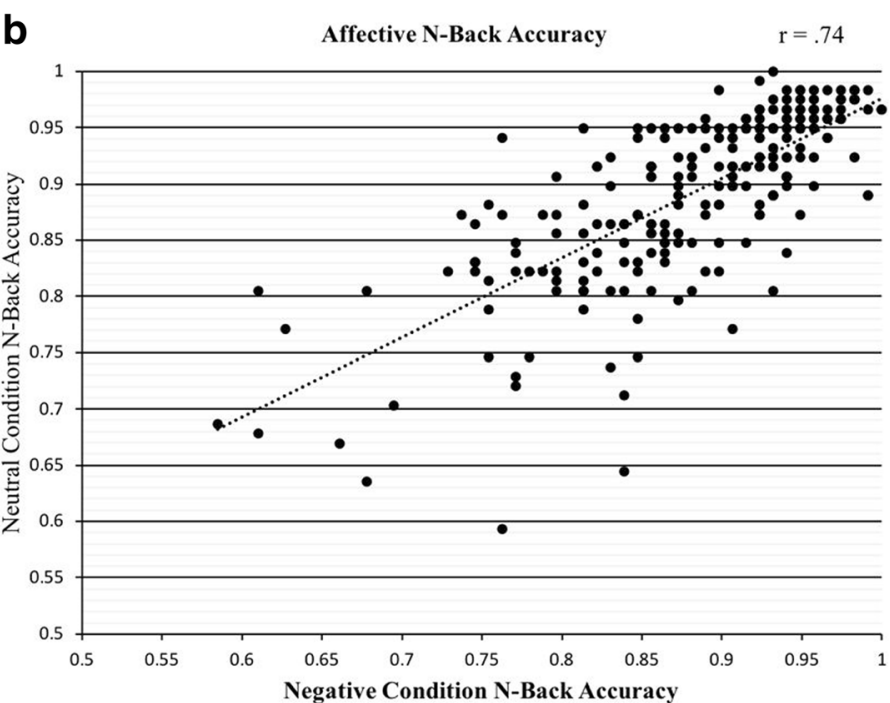

d Willingness to Wait Tendency

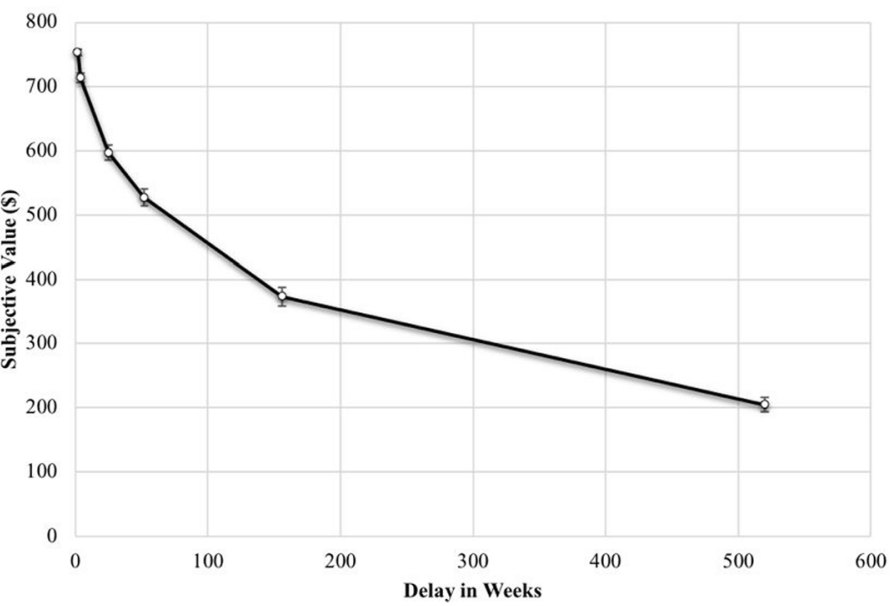

and accuracy was correlated within an individual (B). Willingness to work (percentage of hard choices) significantly differed by reward probability conditions $(\mathbf{C})$. In the delay-discounting task, the subjective value differed by duration of wait (D)

In the EEfRT, 16 participants were excluded for not completing at least 50 trials, which resulted in too few trials in each task condition to properly assess behavior (see Treadway et al., 2009, for more details). An additional ten participants were excluded on the basis of the percentage of completed trials-that is, by making enough button presses. Participants were excluded if they were more than three standard deviations below the group mean percentage of completed trials $(M=95.2 \%, S D=10.8 \%)$. Among the individuals who were excluded on this basis, their mean percentage of trials completed was $46.5 \%(S D=16.8 \%)$.

\section{Analytic strategy}

The participant demographics and study variables $(N$-back accuracy, DDT $k$, and EEfRT percentage of hard choices) 
were examined for site differences. Follow-up analyses did not show any significant impact of site on the size or direction of any effects. All relevant within-task conditions were examined to ensure that the conditions differed from each other as expected. Specifically, we expected a significant difference in accuracy between $N$-back conditions, such that the negative condition would have significantly reduced accuracy. Additionally, we expected that as the length of the delay increased, an individual's willingness to wait (or the subjective value of the reward) would decrease. Finally, we expected that as the probability of reward receipt increased, an individual's willingness to work, defined as the percentage of hard choices made, would also increase.

Two separate sets of path analyses examined the relationship between individual differences in executive function ability on hot and cold EF tasks (i.e., in neutral $N$-back and negative $N$-back accuracy) and willingness to wait or to work across reward conditions. In each of these path analyses, executive function ability on a hot and on a cold EF task (neutral $N$-back, negative $N$-back accuracy) was related to the six delay periods in willingness to wait and to the three levels of probability in willingness to work $(12 \%, 50 \%, 88 \%)$. Path analyses were specified to examine the relative fit of competing theoretical models.

In the willingness- to -wait path analyses, we compared three path models that described the relation between executive function and willingness to wait. This approach allowed us to fully examine all levels of both the $N$-back and DDT tasks. The first model constrained the relations among the task conditions to be equal for the six levels of the willingness- to -wait task ( 2 weeks, 1 month, 2 months, 6 months, 1 year, 3 years, or 10 years) and the two levels of the EF task (negative and neutral), to assess the correlation between willingness to wait and executive function averaged across the different levels of both constructs (see Fig. 3A). The second model allowed the relation of executive function with willingness to wait to vary across the six levels of willingness to wait, while constraining the associations of the two types of EF tasks to be equal to each other within a condition (see Fig. 3B). The third model allowed the relation between willingness-to-wait conditions and $\mathrm{EF}$ tasks to vary across the two types of EF tasks, while constraining the associations of the six levels of willingness to wait to be equal to each other (see Fig. 3C). In the final model, the 12 associations among the six levels of willingness to wait and the two types of executive function were all allowed to vary freely (see Fig. 3D).

In the willingness-to-work analyses, we compared three path models that described the relation between executive function and willingness to work. This approach allowed us to fully examine all levels of both the $N$-back and EEfRT tasks. The first model constrained the relations among the task conditions to be equal for the three levels of willingness to work $(12 \%, 50 \%, 88 \%)$ and the two levels of the EF task (negative, neutral). The first model assessed the correlation between willingness to work and executive function, averaged across the different levels of both constructs (see Fig. 4A). The second model allowed the relation of executive function with willingness to work to vary across the three levels of willingness to work, while constraining the associations of the two types of EF tasks to be equal to each other within a condition (see Fig. 4B). The third model allowed the relations of willingness-to-work conditions with the EF tasks to vary across the two types of executive function, while constraining the associations of the three levels of willingness to work to be equal to each other (see Fig. 4C). In the final model, the six associations among the three levels of willingness to work and the two types of executive function were all allowed to vary freely (see Fig. 4D).

Comparisons among these models provided tests of the main effects of the different levels of willingness to wait or to work on the EF tasks and their interaction. If Model B fit better than Model A in these figures, this would indicate that the association between EF tasks and willingness to wait or to work varied as a function of the main effect of the different levels of willingness to wait or to work. If Model C fit better than Model A, this would indicate that the association between executive function and willingness to wait or to work varied as a function of the main effect of the different types of EF tasks (e.g., executive function ability would be a stronger predictor of willingness to work in one of the EF tasks than in the other). If Model $\mathrm{D}$ fit better than either Model B or C, that would indicate that there was a significant interaction between level of willingness to wait or to work and EF tasks.

Power for the comparisons between correlational models described above was estimated using the conventions and Table 4.3.1 from Cohen (1988) for testing the differences between correlations. ${ }^{1}$ Accordingly, the present study had a power of .30 to detect a small effect size $(q=0.1), .96$ to detect a medium effect size ( $q=0.3$ ), and 1.0 to detect a large effect size $(q=0.5)$. Alternatively, the sample sizes required to have an achieved power of .80 would have been greater than 1,000 for a small effect size, 140 for a medium effect size, and 52 for a large effect size.

\footnotetext{
${ }^{1}$ An alternative to our comparisons of the more complex models that allowed the paths to differ from each other versus the more constrained models that constrained the paths to equal each other would have been to conduct Wald tests on the constraints in a single model. For example, in Model C, in which the $N$-back neutral effect was allowed to differ from the $N$-back negative effect, we could have added a Wald test command to test whether these two associations equaled each other. If that test were significant, it would indicate that the two associations were different. That is, it would indicate the same thing as when Model C, which as we said allowed $N$-back neutral and $N$-back negative to have different effects, provided a significantly better fit than did Model A, which constrained these two effects to equal each other. Thus, it can be seen that our approach is equivalent to testing the differences between correlations.
} 
a

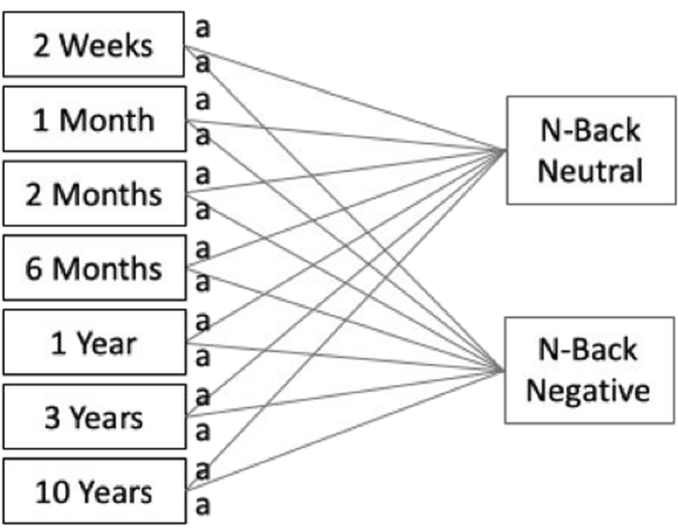

C

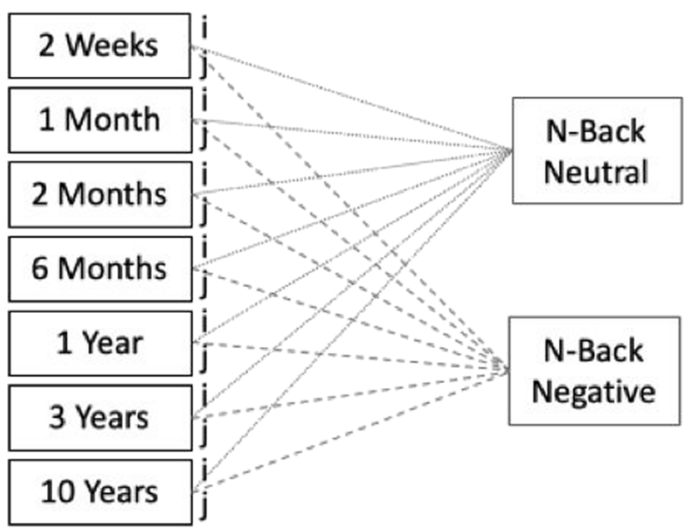

Fig. 3 Willingness- to- wait path models: (A) Model in which all relations are constrained to be one equal value (a). (B) Model that constrains each of the six time delay conditions to relate to the negative and neutral word $N$-back tasks with equal value $(\mathrm{b}-\mathrm{h})$, but where the variables b-h can vary from one another. $(\mathbf{C})$ Model that constrains the

\section{Results}

\section{Participants}

Group differences in demographic characteristics were examined with independent $t$ tests and chi-square tests, summarized in Table 1. We found a significant but small site difference in terms of age: UCLA, $M=19.04, S D=0.65$; NU, $M=19.26, S D$ $=0.64 ; t(227)=2.53, p=.012, d=0.34$. Analyses explored the possible contribution of age to the model, which showed no significant effect or interaction in any of the models, $p \mathrm{~s}>.28$. There was no significant difference in sex across sites: UCLA, $66.2 \%$ female; NU, $64.6 \%$ female; $\chi^{2}(2)=0.90, p=.64$.

\section{Cross site comparison}

Site of data collection had no impact on affective $N$-back accuracy for either the neutral conditions (UCLA, $M=89.5 \%, S D=$ $0.08 \%$; NU, $M=88.8 \%, S D=0.07 \%), t(227)=0.75, p=.93$, or b

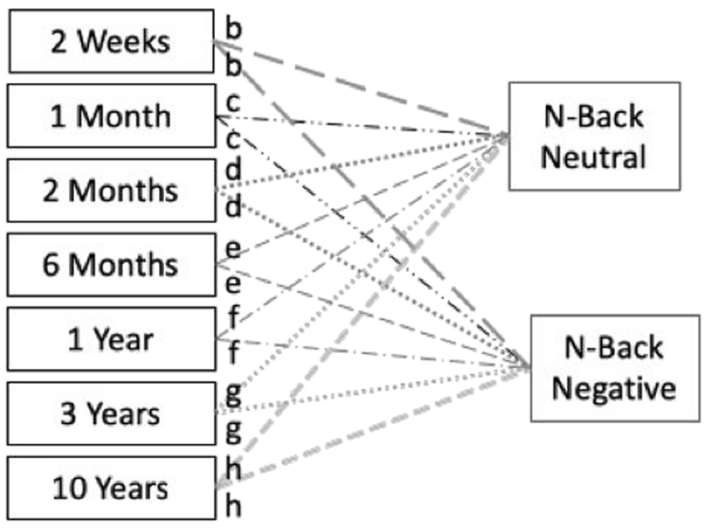

d

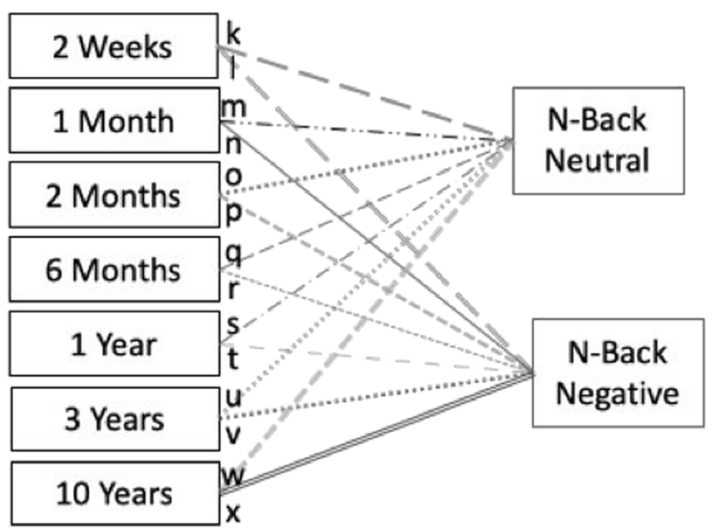

relations of the neutral $N$-back to all DDT conditions (solid lines) to be of equal value (i) and that constrains the relations of the negative $N$-back to all DDT conditions (dashed lines) also to be of equal value (j), but in which $i$ and $j$ can vary from one another. (D) Model in which all paths ( $k$ $\mathrm{x})$ can vary from one another

the negative conditions (UCLA, $M=88.8 \%, S D=0.08 \%$; NU, $M=87.4 \%, S D=0.07 \%) ; t(227)=1.31, p=.35$. There was no significant site difference in willingness to wait, in terms of DDT hyperbolic $k$ (UCLA, $M=0.16, S D=0.05$; NU, $M=$ $0.23, S D=0.22), t(227)=1.29, p=.20, d=0.50$. We also observed no significant effect of site on willingness to work, as measured by the percentage of hard choice responses (UCLA, $M=35.4 \%, S D=16.7 \%$; NU, $M=39.3 \%, S D=14.2 \%), t(227)$ $=1.88, p=.07$. The potential effect of site on hard choices was also examined by probability-of-reward conditions $(12 \%, 50 \%$, or $88 \%$ ). A repeated measures multiple regression demonstrated no significant effect of site, $F(227,1)=3.45, p=.07$, nor was there a significant interaction of reward probability across sites, $F(227,1)=1.91, p=.15($ see Table 1$)$.

\section{Task conditions}

There was a very small but significant difference in affective $N$-back task performance between negative and neutral 

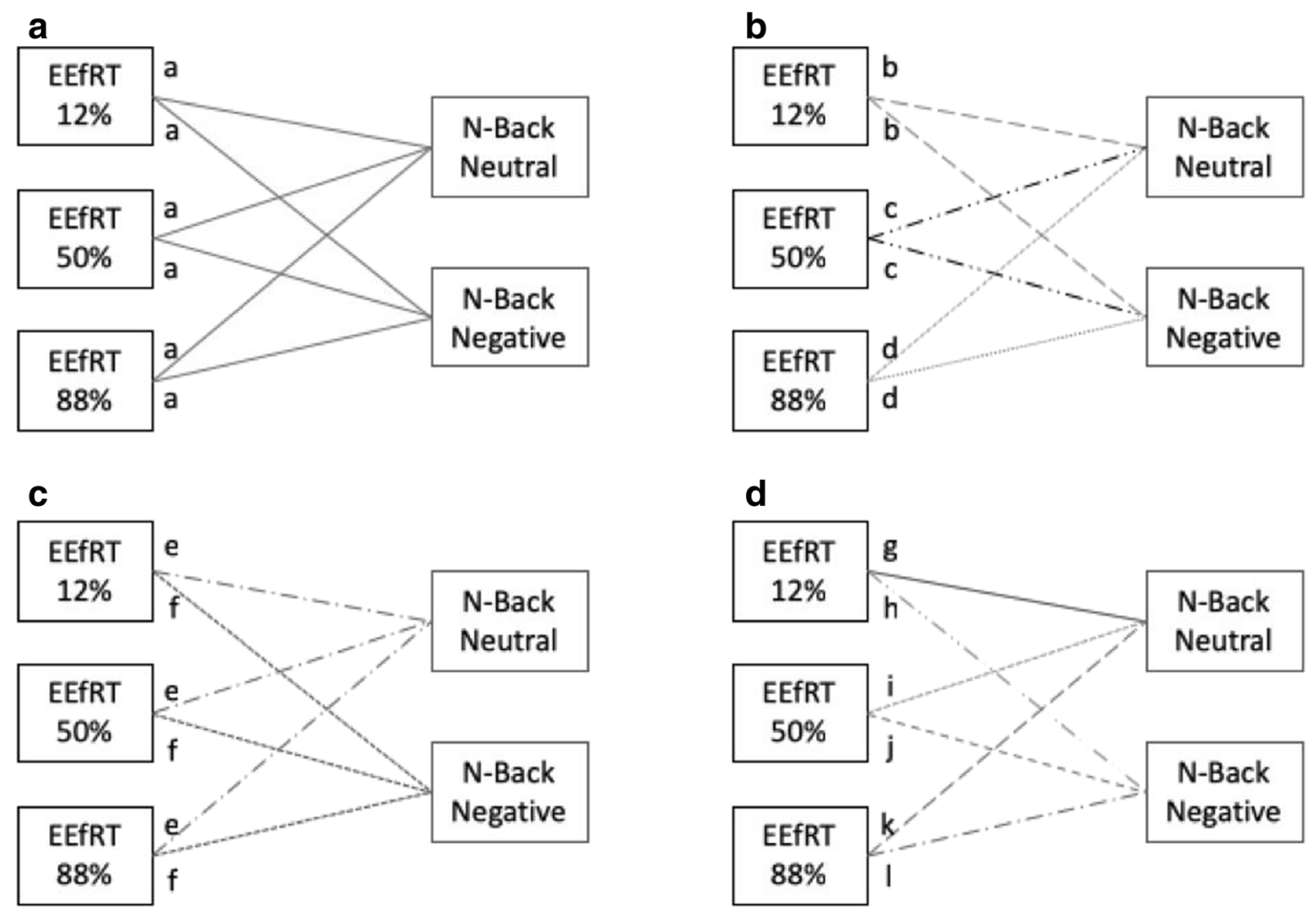

Fig. 4 Willingness- to- work path models: (A) Model in which all relations are constrained to have one equal value (a). (B) Model that constrains the relations of the $12 \%$ probability-of-reward condition (b), the $50 \%$ probability-of-reward condition (c), and the $88 \%$ probability-ofreward condition (d) to each be of equal value across the negative and neutral word $n$-back conditions, but where b, c, and d can vary from one

conditions, such that neutral word accuracy $(M=89.1 \%$, $S D=7.5 \%)$ was significantly greater than negative word accuracy $(M=88.0 \%, S D=7.9 \%), t(227)=2.94, p=$ $.004, d=0.14$ (see Fig. 2A). Affective $N$-back task accuracies in the hot (negative content) and cold (neutral content) conditions were, however, highly positively correlated, $r(229)=.74, p<.001$ (see Fig. 2B). For the willingness-towork task, we found a significant difference in the percentages of hard choices across the probability-of-reward conditions, $12 \%, 50 \%$, and $88 \%, F(2,456)=564.72, p<.001$, $\eta_{\mathrm{p}}{ }^{2}=.71$ : When there was only a $12 \%$ chance of receiving a reward, participants selected a lower proportion of the harder, more effortful trials $(M=11.0 \%, S D=1.0 \%)$ than when there was a $50 \%(M=41.6 \%, S D=1.6 \%)$ or an $88 \%(M=60.5 \%, S D=1.4 \%)$ chance of reward, Fig. $2 \mathrm{C}$. The $50 \%$ probability-of-reward condition also had a significantly lower number of hard work choices than did the $88 \%$ chance of reward, $p<.001$. During the willingnessto-wait task, the subjective value of $\$ 800$ significantly differed over the six delay periods, $F(1692,5)=224.00, p<$ $.001, \eta_{\mathrm{p}}{ }^{2}=.922$, and all delay periods were significantly different, $p<.001$; see Fig. 2D. It should be noted that, although both the DDT and EEfRT reflect cognitive

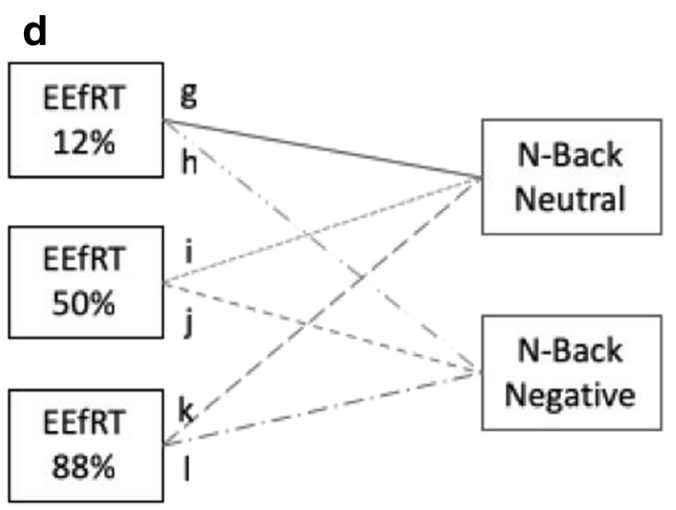

another. (C) Model that constrains the relations of the neutral $n$-back task to be of equal value (e) across EEfRT conditions, and that constrains the relations of the negative $n$-back task also to be of equal value (f) across EEfRT conditions, but where e and $\mathrm{f}$ can vary from one another. (D) Model in which all paths ( $\mathrm{g}-1)$ can vary from one another

features of reward tendencies (willingness to wait or to work for reward), these general tendencies were not significantly correlated, $r(226)=-.03, p=.66$.

\section{Executive functioning and willingness to wait by reward condition}

To examine the relationships between individual differences in executive function ability in the hot and cold EF tasks (neutral and negative $\mathrm{N}$-back accuracy, respectively) and the six reward delay conditions in the willingness-to-wait task (2 weeks, 1 month, 6 months, 1 year, 3 years, and 10 years), we compared the four path models described above. Model B did not provide a significantly better fit than Model A, $\chi^{2}(5)=10.31, p=.07$, but Model $\mathrm{C}$ did provide a significantly better fit than Model A, $\chi^{2}(1)=5.32, p=$ .02 . Model $\mathrm{D}$, however, provided a significantly better fit than Model C, $\chi^{2}(10)=23.16, p=.01$. Thus, despite Model $\mathrm{C}$ being accepted, since it fit significantly better than Model A, Model D fit significantly better than Model C. Model D was thus accepted as the model with the best fit. See Table 2. 
Table 2 Path analysis model fit comparisons and the relationship between executive function and willingness to wait and willingness to work for reward, by reward conditions

\begin{tabular}{|c|c|c|c|c|}
\hline Willingness to wait & & $\chi^{2}$ Difference & $\chi^{2} d f$ Difference & $p$ Value \\
\hline \multicolumn{5}{|c|}{ SEM model comparison } \\
\hline Model A & Model B & 10.31 & 5 & .07 \\
\hline Model A & Model C & 5.32 & 1 & $.02^{*}$ \\
\hline \multirow[t]{2}{*}{ Model C } & Model D & 23.16 & 10 & $.01^{*}$ \\
\hline & \multicolumn{2}{|c|}{ Neutral $N$-back accuracy } & \multicolumn{2}{|c|}{ Negative $N$-back accuracy } \\
\hline Delay condition & $\beta$ & $p$ Value & $\beta$ & $p$ Value \\
\hline 2 weeks & -.11 & .25 & .28 & $.003^{* *}$ \\
\hline 1 month & -.16 & .084 & .35 & $<.001^{* *}$ \\
\hline 6 months & -.27 & $.004^{* *}$ & .45 & $<.001^{* *}$ \\
\hline 1 year & -.18 & .06 & .27 & $.006^{* *}$ \\
\hline 3 years & -.18 & .068 & .287 & $.005^{* *}$ \\
\hline 10 years & -.21 & $.032^{*}$ & .18 & .064 \\
\hline Willingness to work & & $\chi^{2}$ Difference & $\chi^{2} d f$ Difference & $p$ Value \\
\hline \multicolumn{5}{|c|}{ SEM model comparison } \\
\hline Model A & Model B & 21.03 & 2 & $<.0001^{* *}$ \\
\hline Model A & Model C & 0.33 & 1 & .57 \\
\hline \multirow[t]{2}{*}{ Model B } & Model D & 1.13 & 3 & .77 \\
\hline & \multicolumn{4}{|c|}{$N$-back accuracy (general) } \\
\hline Probability condition & $\beta$ & $p$ Value & & \\
\hline $12 \%$ & -.07 & .33 & & \\
\hline $50 \%$ & .40 & $>.001$ & & \\
\hline $88 \%$ & .29 & .003 & & \\
\hline
\end{tabular}

** Significant $p$ values $.005->.001{ }^{*}$ Significant $p$ values $.01-.006$. DDT = delay-discounting task, EEfRT = "effort expenditure for reward" task

\section{Willingness to Work: Best-fit Model D description}

In Model D, the "2-week delay of reward" condition was significantly related to differences in executive function ability in the negative, hot EF task $(\beta=.28, p=.003)$, but not to differences in executive function ability in the neutral, cold EF task $(\beta=-.11, p=.25)$. The "1-month delay of reward" condition was significantly related to differences in executive function ability in the negative, hot EF task $(\beta=.35, p<.001)$, but not differences in executive function ability in the neutral, cold $\mathrm{EF}$ task $(\beta=-.16, p=$ $.08)$. The " 6 -month delay of reward" condition was significantly related to differences in executive function ability in both the negative, hot $\mathrm{EF}$ task $(\beta=.45, p<.001)$, and in the neutral, cold EF task $(\beta=-.27, p=.004)$. The "1-year delay of reward" condition was significantly related to differences in executive function ability in the negative, hot EF task $(\beta=.27, p=.006)$, but not to executive function ability in the neutral, cold EF task $(\beta=-.18, p=.06)$. The "3-year delay of reward" condition was significantly related to differences in executive function ability in the negative, hot $\mathrm{EF}$ task $(\beta=.28, p=.005)$, but not to executive function ability in the neutral, cold EF task $(\beta=-.17, p=$ $.07)$. The "10-year delay of reward" condition was not significantly related to differences in executive function ability in the negative, hot EF task $(\beta=.18, p=.06)$, but was significantly related to executive function ability in the neutral, cold EF task $(\beta=-.21, p=.03)$. See Fig. 5C.

Follow-up analyses examined the possibility of a quadratic relationship between executive function ability in the negative, hot EF task across the delay conditions. In this model, three factors were created, corresponding to the trajectory of DDT performance as a function of the delay interval - an intercept factor that corresponds to the level of DDT performance at the 6-month delay, a linear factor that represents linear changes as a function of the delay interval, and a quadratic factor that represents positive, U-shaped, or negative, inverted-U-shaped, changes as a function of the delay interval. Then, each factor was regressed on the differences in executive function ability in the hot EF task (since performance in the neutral context showed a linear increase). The regression of the intercept factor on the differences in executive function ability in the hot EF task was significant $(p<.001)$, as was the regression of the quadratic factor on the differences in executive function ability in the hot EF task $(p<.001)$, but the linear factor was not significantly related to the differences in executive function ability in the hot EF task $(p=.06)$. See Fig. 5C. 


\section{a Quartile Plot of Willingness to Wait}

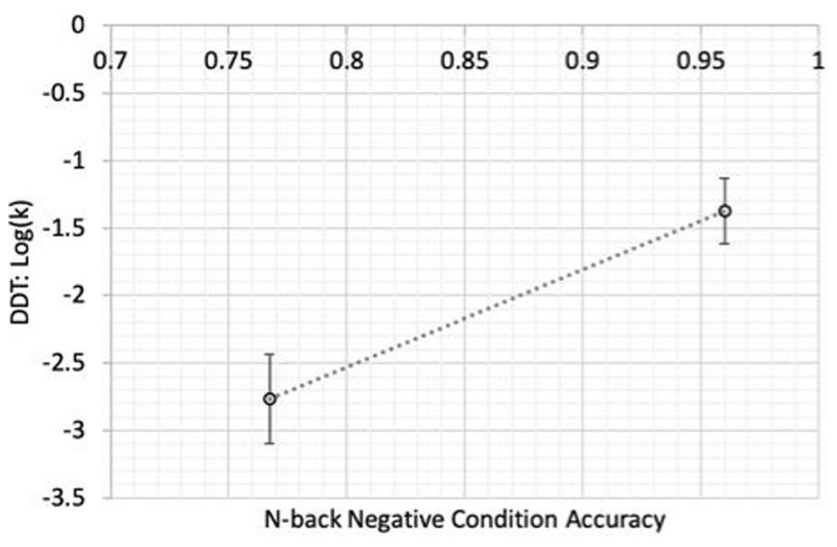

C Relationship between N-Back Accuracy Delay Discounting

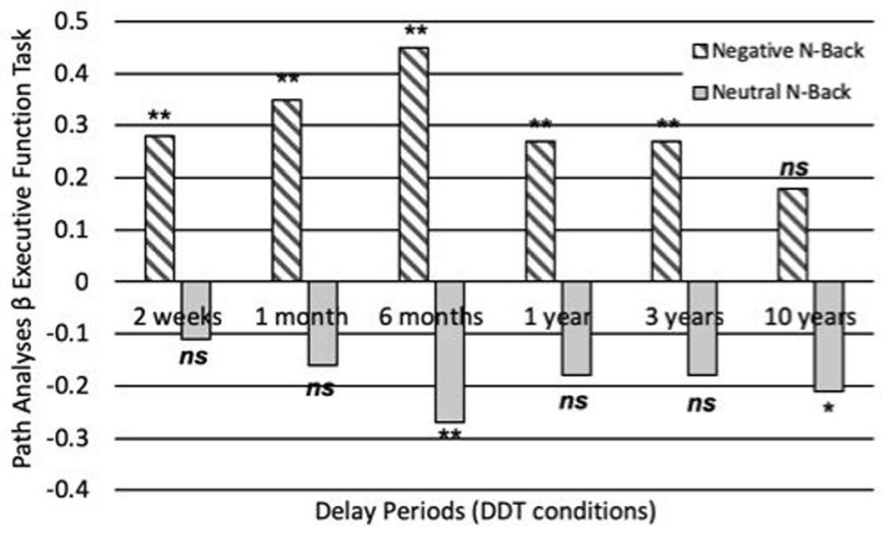

Fig. 5 Relations of affective $N$-back task accuracy to the delaydiscounting task (DDT; A, C) and to the "effort expenditure for reward" task (EEfRT; B, D): (A) Quartile split (25th vs. 75th) comparing willingness to wait to executive function. (B) Quartile split (25th vs. 75th) comparing willingness to work to executive function. (C) Plot of beta weights

\section{Executive functioning and willingness to work by reward condition}

To examine the relationships between individual differences in executive function ability on the hot and cold EF tasks (neutral and negative $N$-back accuracy, respectively) and the three levels of probability for willingness to wait $(12 \%, 50 \%, 88 \%)$, we compared the four path models described above. Model B provided a significantly better fit than Model A, $\chi^{2}(2)=21.03, p<$ .001 . Model $\mathrm{C}$, however, did not provide a significantly better fit than Model A, $\chi^{2}(1)=0.33, p=.57$. Model D likewise did not provide a significantly better fit than Model B, $\chi^{2}(3)=1.13, p=.77$ (see Table 2). Thus, Model B was accepted, since it fit significantly better than Model A and was more parsimonious than Model D, which did not fit significantly better than Model B. As a result, Model B was accepted as the model of best fit (see Table 2).

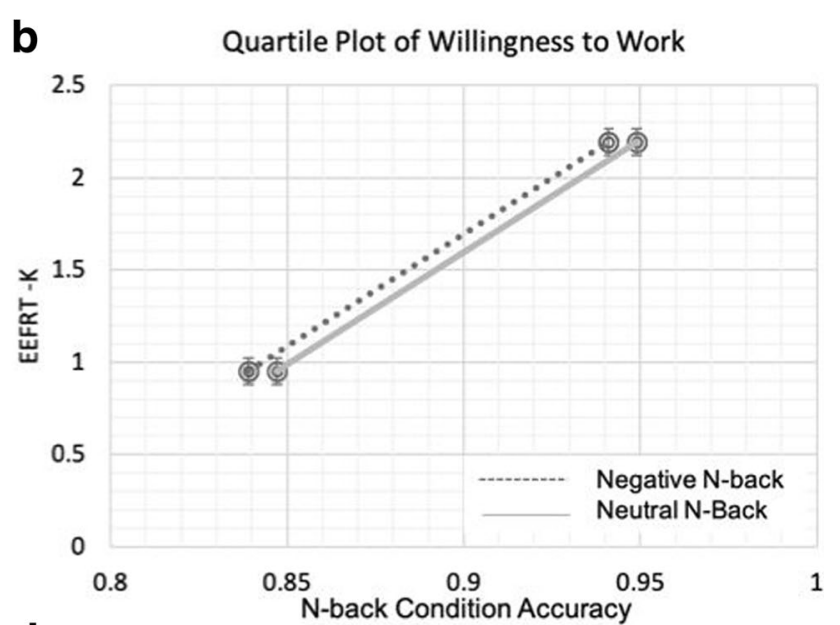

d

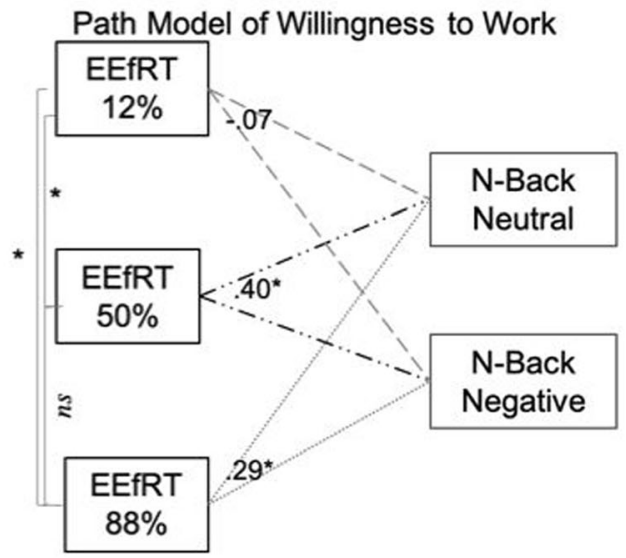

from path analyses characterizing the relationship between willingness to work and executive function ability over delay conditions. (D) Path model with beta weights characterizing the relationship between willingness to wait and executive function ability over delay conditions

\section{Willingness to Wait: Best-fit Model B description}

In Model $\mathrm{B}$, the $12 \%$ probability-of-reward condition did not significantly relate to executive function $(\beta=-.07, p=$ .33 ); this $\beta$ weight refers to path $\mathrm{b}$ in Fig. $4 \mathrm{~B}$. The $50 \%$ probability-of-reward condition was significantly related to executive function $(\beta=.40, p<.001)$; this $\beta$ weight refers to path $\mathrm{c}$ in Fig. 4B. The $88 \%$ probability-of-reward condition was significantly related to executive function $(\beta=$ $.29, p=.003$ ); this $\beta$ weight refers to path $\mathrm{d}$ in Fig. 4B. Follow-up comparisons of unstandardized coefficients revealed that willingness to work was related differently to average executive function accuracy in the two EF tasks, such that the $12 \%(\beta=-.07,95 \% \mathrm{CI}=-.19$ to .05$)$ probability of reward was significantly different from the $50 \%(\beta=.40,95 \% \mathrm{CI}=.22$ to .58$)$ or the $88 \%(\beta=.13$, $95 \% \mathrm{CI}=.10$ to .44$)$ probability of reward. The $50 \%$ and $88 \%$ probability-of-reward conditions did not differ in their relationships to executive function. See Fig. 5D. 


\section{Discussion}

In the present study we assessed the relationships between executive function ability on hot and cold EF tasks and willingness to work and willingness to wait for reward. In line with predictions and past research, willingness to wait for rewards was positively related to individual differences in executive function ability on a hot EF task. Surprisingly, executive function ability on a cold EF task showed a small, but significant, negative relationship with willingness to wait at 6month and 10-year delays. Finally, the association between executive function ability on a hot EF task and willingness to wait for reward varied significantly across the delay conditions in a quadratic fashion, and the relationship was strongest at a 6-month delay. Greater executive function ability was positively related to a greater willingness to work, as expected. Contrary to our hypothesis, there was no evidence of differential associations between willingness to work and executive function ability in hot and cold EF task conditions. In willingness-to-work analyses, greater executive function ability in both hot and cold EF tasks was related to a greater willingness to work in contexts where the probability of reward was likely $(88 \%)$ or highly uncertain $(50 \%)$, as compared to when reward was unlikely $(12 \%)$. This variability by reward condition suggests that executive function ability in both hot and cold EF tasks reflects sensitivity of valuebased decisions to contextual features of the reward condition.

Willingness to wait was positively related to executive function ability in a hot EF task, as expected, and weakly but negatively associated with executive function ability in a cold EF task, contrary to expectations. The specificity of this relationship suggests that individual differences in executive function abilities in a hot EF task may be a critical aspect of willingness to wait, beyond the contribution of executive function ability in a cold EF task. Additionally, this interaction is in line with past research that suggested that the emotional and motivational context of willingness-to-wait decisions might reflect an individual's executive function ability in the face of distracting emotional information (see Zelazo \& Carlson, 2012). The amount of variance in willingness to wait explained by executive function ability in a hot $\mathrm{EF}$ task varied across the reward delay conditions in a quadratic fashion, peaking at 6 months. Individual differences in executive function ability in a hot EF task were not as predictive of performance at immediate delays as in the other delay conditions $(\beta \mathrm{s}=.28-.35)$.

This quadratic relationship was in contrast to our prediction that the amount of variance explained by executive function ability in a hot EF task would reflect the general preference for more immediate rewards - for instance, a positive linear relationship. Alternatively, if resisting the temptation of immediate rewards were a direct reflection of executive function ability in a hot $\mathrm{EF}$ task, then the relationship of subjective value to executive function ability on a hot EF task would reflect the subjective discounting hyperbolic curve. In contrast to these hypotheses, the unexpected quadratic relationship implies that executive function ability in a hot EF task does not simply reflect a resistance of general tendencies toward immediate reward (consistent with a positive linear relationship), nor the pragmatic discounting of the reward based on delay length (consistent with a hyperbolic relationship). This quadratic relationship also emphasizes that willingness-to-wait tasks should not be used as a primary metric of executive function ability in a hot context, because this would fail to account for the complex contribution to executive function ability in a hot context of different delay conditions. In fact, this quadratic relationship highlights the consideration of other factors that might also influence willingness to wait.

One interpretation of this quadratic relationship may be that executive function ability accounts for the most variance in willingness to wait at a 6 -month delay $(\beta=.45)$ because this is a distant, but imaginable, future in which it may be beneficial to ignore immediate reward for a larger future reward. In contrast, executive function ability in the face of negative distracting delay information may be less important for very short delays ( 2 weeks or 1 month), when rewards are more immediate. Executive function ability in the face of negative distracting information may also be less important for very long delays, because very few people will be able to overcome the costs of a 1- to 10-year delay in pursuit of a reward. Alternatively, it is also possible that choosing a delayed reward might not always be the best choice. As a result, variance in choosing to wait for a reward may not be accounted for by executive function ability in a hot EF task because it does not reflect the optimal cognitive choice in the face of distracting emotional information - for example, at a very long delay (a 1- to 10-year delay), where the reward might not be worth the wait at such a long delay. In such a case, choosing to wait for a reward might not be the best choice, and as a result, individual differences in a hot EF task would not be as predictive for suboptimal choices ( $\beta \mathrm{s}=.18-.27)$. The present finding may contradict the perspective that resisting the temptation of an immediate reward is a direct reflection of executive function ability in a hot EF task. Instead, the variability of the relationship between DDT and performance in a hot EF task suggests that delay-discounting tasks are not a sufficient primary measure of executive function in a hot EF task, but might reflect the cognitive strategy being employed.

Willingness-to-work tendencies were positively related to executive function ability in general, as expected, but this relationship did not vary significantly across hot and cold EF tasks, contrary to our predictions. The amount of variance in willingness to work explained by executive function ability did vary across reward probability conditions. Higher willingness to work for a $50 \%$ and an $88 \%$ chance of reward was positively related to greater executive function ability, as expected. However, when there was only a $12 \%$ chance of 
reward, the relationship between individual differences in executive function ability and willingness to work was significantly less positive. The $12 \%$ probability-of-reward condition, in which the cost of work was high and the chance of reward unlikely, might require less executive function ability. This difference between reward probability conditions also suggests that the low-likelihood (12\%) trials were not as relevant to individual differences in executive function ability as the uncertain or likely (50\% and $88 \%$ ) trials. This difference between reward probabilities is in line with our prediction that executive function ability would account for more of the variance in individual differences as likelihood of reward increased, although there were no significant differences between the uncertain $(50 \%)$ and likely $(88 \%)$ conditions. Finally, the main effect of willingness-to-wait task conditions suggests that executive function ability in hot EF tasks is particularly important for decisions in which there is a reasonable likelihood of reward. In other words, high executive function ability may be useful to sustain performance when the likelihood of reward is high, but when there is a low chance of reward, individuals are less motivated to work for the reward, regardless of individual differences in executive function ability.

The present study focused on how individual differences in executive function ability in hot and cold tasks relate to valuebased decision making. However, future studies should consider additional features of executive function. First, some research has suggested that individual differences in executive function ability on a hot EF task may be impacted differently by positive or negative valence content (Joormann \& Vanderlind, 2014). Given that both positively valenced rewards and negatively valenced costs are involved in valuebased decisions, individual differences in executive function ability in both positive and negative contexts might provide unique contributions to decisions regarding whether to wait or work for rewards. The present study was not equipped to test such a possibility, since the executive-functioning tasks consisted of negative and neutral, but not positive, EF tasks. Nevertheless, future research should examine the potential impact of positive valence.

Future studies should also consider both the unity and diversity of executive function. The present study relied on the $\mathrm{N}$-back task, which taps a general factor of executive function, rather than any one specific facet (Jaeggi, Buschkuehl, Perrig, \& Meier, 2010; Miller, Price, Okun, Montijo, \& Bowers, 2009). As a result, the present study is unable to speak to the relative contributions of specific facets of executive function to value-based decision making. However, a number of facets of executive function might be important for future research on willingness to work and to wait. Specifically, Miyake et al. (2000) highlighted the importance of both a general factor and specific facets (i.e., shifting, updating, and inhibition) to executive functioning. Although the distinguishable processes underlying executive function are highly interrelated, they may provide distinct contributions to willingness-to-wait and willingness-to-work tendencies. For example, inhibition blocks out irrelevant information during value-based decision making, and thus deficits in inhibition might lead to impulsive decisions (e.g., less willingness to wait). Similarly, updating abilities might help individuals properly weigh the subjective value of options based on the changing costs and benefits, and deficits in updating could result in less willingness to wait or work for rewards. Finally, shifting is the ability to move swiftly between multiple actions, and greater shifting abilities might better equip individuals to consider the multiple outcomes of any given value-based decision making. Future studies should examine the extent to which each facet of executive function ability relates to value-based decision making (Miyake et al., 2000).

The distinct relationship of executive function ability with different choice tendencies toward willingness to wait and to work might reflect the differences in the neural substrates underlying these decisions. In the present study we found that willingness-to-wait choice tendencies are related to executive function ability in the face of negative information. From past research, we know that willingness-to-wait choices are particularly related to activity in the ventral medial prefrontal cortex (Massar et al., 2015; Prévost et al., 2010; Rudebeck et al., 2006; Seaman et al., 2018). This ventral medial prefrontal cortex activity during willingness-to-wait choices might also be related to executive function ability in the face of distracting, emotional stimuli. In contrast, the present study showed that willingness-to-work choices are related to executive function ability, regardless of whether the EF task is hot or cold. Past research has also demonstrated that willingness to work is related to anterior cingulate cortex activity (Prévost et al., 2010; Rudebeck et al., 2006; Seaman et al., 2018). It is possible that the anterior cingulate cortex activity during willingness-to-work choices might also be related to overall executive function ability. Unfortunately, one limitation of this behavioral study is that it was not able to directly examine the relationship of activity in these regions to individual differences in executive function in emotional contexts. Future studies should examine the neural mechanisms underlying the associations between executive function and value-based decision making observed in the present study.

Another potential limitation is that there might be relevant differences in the value-based decision-making tasks. Particularly, the willingness-to-wait task asked participants about hypothetical monetary rewards at hypothetical delays. In contrast, the willingness-to-work task asked participants to expend real effort for real monetary rewards. Some research has suggested that hypothetical rewards are less salient than real monetary rewards, and that this difference results in distinct effects on value-based decision-making behavior and related brain activity (Xu et al., 2018). As a result, one might 
expect that the willingness-to-wait task would be unrelated to executive function ability in a hot EF task, because the hypothetical rewards and costs would be less emotional and distracting. Similarly, if the willingness-to-wait task was not sufficiently salient, we might expect that it would reflect executive function ability in a cold EF task, because the task contained neutral, hypothetical information. Our findings, however, are inconsistent with this concern. In fact, we found that a willingness to wait for reward was more related to executive function ability in a hot than in a cold EF task. It is noteworthy that previous research has also observed that real and hypothetical rewards do not differentially impact delaydiscounting tendencies (Johnson \& Bickel, 2002; Lagorio \& Madden, 2005). It is still possible that the use of hypothetical rewards in the present study underestimated the impact of individual differences in executive function ability in hot $\mathrm{EF}$ tasks on willingness to work. Therefore, future studies should explore how individual differences in executive function ability in the face of distracting emotional or neutral information relate to value-based decision making for both real and hypothetical costs and rewards.

In conclusion, our results suggest that a willingness to wait and a willingness to work relate to executive-functioning tendencies. Greater executive function ability in a hot EF task was more related to a greater willingness to wait than was executive function ability in a cold EF task. In contrast, better executive function ability in both hot and cold EF tasks was related to a greater willingness to work, with no evidence of differential associations between individual differences in executive-functioning ability based on whether the EF task was hot or cold. We also found that the strength of the relationship between individual differences in executive function ability varied across value-based decision conditions. A greater willingness to wait for reward was more strongly related to executive function ability in a hot EF task when the reward was in 6 months than with either more immediate (1 week, 1 month) or more distant (1 year, 3 years, 10 years) delays. Similarly, we found that a greater willingness to work for reward was more related to executive functioning when reward was uncertain $(50 \%)$ or likely $(88 \%)$ than when the reward was unlikely $(12 \%)$. These results both emphasize the importance of individual differences in executive function ability during value-based decision making, and they provide evidence that the importance of executive function ability may vary with cost and benefit features of the decision. Collectively, our results suggest that individual differences in executive function ability in hot tasks may be particularly important for willingness to wait, but that executive function ability in general is important for willingness to work. Therefore, improved executive function ability in the face of distracting emotional information may be of particular benefit in willingness-to-wait decisions, such as increasing saving for retirement (Odum, 2011a) or reducing problematic behaviors such as drug abuse, obesity, and gambling (Daugherty \& Brase, 2010; Kirby et al., 2005; Odum, 2011a). In contrast, strategies to improve executive function in general may benefit willingness-to-work decisions that are relevant to clinical disorders such as depression and schizophrenia (Barch et al., 2014; Treadway et al., 2009).

Author note The data for the experiment reported here are available upon request through the BrainMAPD Project website; the experiment was not preregistered, and all of the R and MPLUS analysis scripts are available at github.com/katedamme.

\section{References}

Ahn, W.-Y., Rass, O., Fridberg, D. J., Bishara, A. J., Forsyth, J. K., Breier, A., ... O'Donnell, B. F. (2011). Temporal discounting of rewards in patients with bipolar disorder and schizophrenia. Journal of Abnormal Psychology, 120, 911-921. https://doi.org/10.1037/ a0023333

Aridan, N., Malecek, N. J., Poldrack, R. A., \& Schonberg, T. (2019). Neural correlates of effort-based valuation with prospective choices. NeuroImage, 185, 446-454. https://doi.org/10.1016/j.neuroimage. 2018.10.051

Arulpragasam, A. R., Cooper, J. A., Nuutinen, M. R., \& Treadway, M. T. (2018). Corticoinsular circuits encode subjective value expectation and violation for effortful goal-directed behavior. Proceedings of the National Academy of Sciences, 115, E5233-E5242.

Banich, M. T. (2009). Executive function: The search for an integrated account. Current Directions in Psychological Science, 18, 89-94.

Barch, D. M., Treadway, M. T., \& Schoen, N. (2014). Effort, anhedonia, and function in schizophrenia: Reduced effort allocation predicts amotivation and functional impairment. Journal of Abnormal Psychology, 123, 387-397. https://doi.org/10.1037/a0036299

Benningfield, M. M., Blackford, J. U., Ellsworth, M. E., Samanez-Larkin, G. R., Martin, P. R., Cowan, R. L., \& Zald, D. H. (2014). Caudate responses to reward anticipation associated with delay discounting behavior in healthy youth. Developmental Cognitive Neuroscience, $7,43-52$.

Bickel, W. K., Jarmolowicz, D. P., Mueller, E. T., Gatchalian, K. M., \& McClure, S. M. (2012). Are executive function and impulsivity antipodes? A conceptual reconstruction with special reference to addiction. Psychopharmacology, 221, 361-387.

Botvinick, M. M., Huffstetler, S., \& McGuire, J. T. (2009). Effort discounting in human nucleus accumbens. Cognitive, Affective, \& Behavioral Neuroscience, 9, 16-27. https://doi.org/10.3758/CABN. 9.1.16

Chatham, C. H., Herd, S. A., Brant, A. M., Hazy, T.E., Miyake, A., O'Reilly, R., Friedman, N. P. (2011). From an executive network to executive control: A computational model of the $n$-back task. Journal of Cognitive Neuroscience, 23, 3598-3619.

Chung, S. H., \& Herrnstein, R. J. (1967). Choice and delay of reinforcement. Journal of the Experimental Analysis of Behavior, 10, 67-74.

Churchwell, J. C., Morris, A. M., Heurtelou, N. M., \& Kesner, R. P. (2009). Interactions between the prefrontal cortex and amygdala during delay discounting and reversal. Behavioral Neuroscience, 123, 1185-1196. https://doi.org/10.1037/a0017734

Cohen, J. (1988). Statistical power analyses for the behavioral sciences (2nd ed.). Hillsdale, NJ: Erlbaum.

Compton, R. J., Arnstein, D., Freedman, G., Dainer-Best, J., Liss, A., \& Robinson, M. D. (2011). Neural and behavioral measures of errorrelated cognitive control predict daily coping with stress. Emotion, 11, 379-390. https://doi.org/10.1037/a0021776 
Croxson, P. L., Walton, M. E., O’Reilly, J. X., Behrens, T. E., \& Rushworth, M. F. (2009). Effort-based cost-benefit valuation and the human brain. Journal of Neuroscience, 29, 4531-4541.

Daugherty, J. R., \& Brase, G. L. (2010). Taking time to be healthy: Predicting health behaviors with delay discounting and time perspective. Personality and Individual Differences, 48, 202-207.

Du, W., Green, L., \& Myerson, J. (2002). Cross-cultural comparisons of discounting delayed and probabilistic rewards. Psychological Record, 52, 479-492.

Friedman, N. P., \& Miyake, A. (2017). Unity and diversity of executive functions: Individual differences as a window on cognitive structure. Cortex, 86, 186-204.

Gatzke-Kopp, L. M., Ram, N., Lydon-Staley, D. M., \& DuPuis, D. (2018). Children's sensitivity to cost and reward in decision making across distinct domains of probability, effort, and delay. Journal of Behavioral Decision Making, 31, 12-24.

Hariri, A. R., Brown, S. M., Williamson, D. E., Flory, J. D., de Wit, H., \& Manuck, S. B. (2006). Preference for immediate over delayed rewards is associated with magnitude of ventral striatal activity. Journal of Neuroscience, 26, 13213-13217. https://doi.org/10. 1523/JNEUROSCI.3446-06.2006

Hinson, J. M., Jameson, T. L., \& Whitney, P. (2003). Impulsive decision making and working memory. Journal of Experimental Psychology: Learning, Memory, and Cognition, 29, 298-306.

Hofmann, W., Schmeichel, B. J., \& Baddeley, A. D. (2012). Executive functions and self-regulation. Trends in Cognitive Sciences, 16, 174-180.

Jaeggi, S. M., Buschkuehl, M., Perrig, W. J., \& Meier, B. (2010). The concurrent validity of the $N$-back task as a working memory measure. Memory, 18, 394-412.

Johnson, M. W., \& Bickel, W. K. (2002). Within-subject comparison of real and hypothetical money rewards in delay discounting. Journal of the Experimental Analysis of Behavior, 77, 129-146. https://doi. org/10.1901/jeab.2002.77-129

Johnson, S. L., Swerdlow, B. A., Treadway, M., Tharp, J. A., \& Carver, C. S. (2017). Willingness to expend effort toward reward and extreme ambitions in bipolar I disorder. Clinical Psychological Science, 5, 943-951.

Joormann, J., \& Quinn, M. E. (2014). Cognitive processes and emotion regulation in depression. Depression and Anxiety, 31, 308-315.

Joormann, J., \& Vanderlind, W. M. (2014). Emotion regulation in depression: The role of biased cognition and reduced cognitive control. Clinical Psychological Science, 2, 402-421.

Kirchner, W. K. (1958). Age differences in short-term retention of rapidly changing information. Journal of experimental psychology, 55(4), 352.

Kirby, K. N., Winston, G. C., \& Santiesteban, M. (2005). Impatience and grades: Delay-discount rates correlate negatively with college GPA. Learning and Individual Differences, 15, 213-222.

Klein-Flügge, M. C., Kennerley, S. W., Saraiva, A. C., Penny, W. D., \& Bestmann, S. (2015). Behavioral modeling of human choices reveals dissociable effects of physical effort and temporal delay on reward devaluation. PLoS Computational Biology, 11, e1004116. https://doi.org/10.1371/journal.pcbi.1004116

Lagorio, C. H., \& Madden, G. J. (2005). Delay discounting of real and hypothetical rewards. III: Steady-state assessments, forced-choice trials, and all real rewards. Behavioural Processes, 69, 173-187.

Laibson, D. I., Repetto, A., Tobacman, J., Hall, R. E., Gale, W. G., \& Akerlof, G. A. (1998). Self-control and saving for retirement. Brookings Papers on Economic Activity, 1998(1), 91-196.

Massar, S. A., Libedinsky, C., Weiyan, C., Huettel, S. A., \& Chee, M. W. (2015). Separate and overlapping brain areas encode subjective value during delay and effort discounting. NeuroImage, 120, 104-113.

Miller, K. M., Price, C. C., Okun, M. S., Montijo, H., \& Bowers, D. (2009). Is the $n$-back task a valid neuropsychological measure for assessing working memory? Archives of Clinical Neuropsychology, 24, 711-717.

Miyake, A., Friedman, N. P., Emerson, M. J., Witzki, A. H., Howerter, A., \& Wager, T. D. (2000). The unity and diversity of executive functions and their contributions to complex "frontal lobe" tasks: A latent variable analysis. Cognitive Psychology, 41, 49-100. https:// doi.org/10.1006/cogp.1999.0734

Odum, A. L. (2011a). Delay discounting: I'm ak, you're ak. Journal of the Experimental Analysis of Behavior, 96, 427-439.

Odum, A. L. (2011b). Delay discounting: Trait variable? Behavioural Processes, 87, 1-9.

Olson, E. A., Hooper, C. J., Collins, P., \& Luciana, M. (2007). Adolescents' performance on delay and probability discounting tasks: Contributions of age, intelligence, executive functioning, and self-reported externalizing behavior. Personality and Individual Differences, 43, 1886-1897.

Patrick, M. E., Blair, C., \& Maggs, J. L. (2008). Executive function, approach sensitivity, and emotional decision making as influences on risk behaviors in young adults. Journal of Clinical and Experimental Neuropsychology, 30, 449-462.

Pessiglione, M., Vinckier, F., Bouret, S., Daunizeau, J., \& Le Bouc, R. (2017). Why not try harder? Computational approach to motivation deficits in neuro-psychiatric diseases. Brain, 141, 629-650.

Pornpattananangkul, N., \& Nusslock, R. (2016). Willing to wait: Elevated reward-processing EEG activity associated with a greater preference for larger-but-delayed rewards. Neuropsychologia, 91, 141-162.

Prévost, C., Pessiglione, M., Météreau, E., Cléry-Melin, M. L., \& Dreher, J. C. (2010). Separate valuation subsystems for delay and effort decision costs. Journal of Neuroscience, 30, 14080-14090.

Quinn, M. E., \& Joormann, J. (2015). Stress-induced changes in executive control are associated with depression symptoms: Examining the role of rumination. Clinical Psychological Science, 3, 628-636.

Rachlin, H., Raineri, A., \& Cross, D. (1991). Subjective probability and delay. Journal of the Experimental Analysis of Behavior, 55, 233 244.

Rangel, A., Camerer, C., \& Montague, P. R. (2008). A framework for studying the neurobiology of value-based decision making. Nature Reviews Neuroscience, 9, 545-556. https://doi.org/10.1038/nrn2357

Rudebeck, P. H., Walton, M. E., Smyth, A. N., Bannerman, D. M., \& Rushworth, M. F. (2006). Separate neural pathways process different decision costs. Nature Neuroscience, 9, 1161-1168. https://doi. org/10.1038/nn1756

Schmeichel, B. J., \& Tang, D. (2015). Individual differences in executive functioning and their relationship to emotional processes and responses. Current Directions in Psychological Science, 24, 93-98.

Seaman, K. L., Brooks, N., Karrer, T. M., Castrellon, J. J., Perkins, S. F., Dang, L. C., \& Samanez-Larkin, G. R. (2018). Subjective value representations during effort, probability and time discounting across adulthood. Social Cognitive and Affective Neuroscience, 13, 449-459.

Shamosh, N. A., DeYoung, C. G., Green, A. E., Reis, D. L., Johnson, M. R., Conway, A. R., . . Gray, J. R. (2008). Individual differences in delay discounting: Relation to intelligence, working memory, and anterior prefrontal cortex. Psychological Science, 19, 904-911.

Shamosh, N. A., \& Gray, J. R. (2008). Delay discounting and intelligence: A meta-analysis. Intelligence, 36, 289-305.

Shead, N. W., \& Hodgins, D. C. (2009). Probability discounting of gains and losses: Implications for risk attitudes and impulsivity. Journal of the Experimental Analysis of Behavior, 92, 1-16.

Snyder, H. R., Miyake, A., \& Hankin, B. L. (2015). Advancing understanding of executive function impairments and psychopathology: Bridging the gap between clinical and cognitive approaches. Frontiers in Psychology, 6, 328. https://doi.org/10.3389/fpsyg. 2015.00328 
Sugiwaka, H., \& Okouchi, H. (2004). Reformative self-control and discounting of reward value by delay or effort. Japanese Psychological Research, 461, 1-9.

Treadway, M. T., Buckholtz, J. W., Cowan, R. L., Woodward, N. D., Li, R., Ansari, M. S., . . Zald, D. H. (2012). Dopaminergic mechanisms of individual differences in human effort-based decision making. Journal of Neuroscience, 32, 6170-6176.

Treadway, M. T., Buckholtz, J. W., Schwartzman, A. N., Lambert, W. E., \& Zald, D. H. (2009). Worth the "EEfRT"? The effort expenditure for rewards task as an objective measure of motivation and anhedonia. PLoS ONE, 4, e6598. https://doi.org/10.1371/journal.pone. 0006598

Wittmann, M., Leland, D. S., \& Paulus, M. P. (2007). Time and decision making: Differential contribution of the posterior insular cortex and the striatum during a delay discounting task. Experimental Brain Research, 179, 643-653.

Wolf, A. M., \& Colditz, G. A. (1998). Current estimates of the economic cost of obesity in the United States. Obesity, 6, 97-106.

Xu, S., Pan, Y., Qu, Z., Fang, Z., Yang, Z., Yang, F., . . . Rao, H. (2018). Differential effects of real versus hypothetical monetary reward magnitude on risk-taking behavior and brain activity. Scientific Reports, 8, 3712. https://doi.org/10.1038/s41598-018-21820-0

Zelazo, P. D., \& Carlson, S. M. (2012). Hot and cool executive function in childhood and adolescence: Development and plasticity. Child Development Perspectives, 6, 354-360.

Publisher's note Springer Nature remains neutral with regard to jurisdictional claims in published maps and institutional affiliations. 